Research Article

\title{
A Smoothing Newton Method with a Mixed Line Search for Monotone Weighted Complementarity Problems
}

\author{
Xiaoqin Jiang $\mathbb{D}^{1}$ and He Huang $\mathbb{D}^{2}$ \\ ${ }^{1}$ Department of Public Basic, Wuhan Technology and Business University, Wuhan 430065, China \\ ${ }^{2}$ College of Management and Economics, Tianjin University, Weijin South Road 192, 300372 Tianjin, China
}

Correspondence should be addressed to He Huang; hhuangtju@163.com

Received 25 February 2020; Accepted 7 May 2020; Published 30 June 2020

Academic Editor: Quanxin Zhu

Copyright (c) 2020 Xiaoqin Jiang and He Huang. This is an open access article distributed under the Creative Commons Attribution License, which permits unrestricted use, distribution, and reproduction in any medium, provided the original work is properly cited.

\begin{abstract}
In this paper, we present a smoothing Newton method for solving the monotone weighted complementarity problem (WCP). In each iteration of our method, the iterative direction is achieved by solving a system of linear equations and the iterative step length is achieved by adopting a line search. A feature of the line search criteria used in this paper is that monotone and nonmonotone line search are mixed used. The proposed method is new even when the WCP reduces to the standard complementarity problem. Particularly, the proposed method is proved to possess the global convergence under a weak assumption. The preliminary experimental results show the effectiveness and robustness of the proposed method for solving the concerned WCP.
\end{abstract}

\section{Introduction}

The weighted linear complementarity problem, introduced by Potra [1], is to find a vector $(s, t, u) \in \mathfrak{R}^{n} \times \mathfrak{R}^{n} \times \mathfrak{R}^{m}$ such that

$$
\begin{aligned}
s, t \geq 0, \\
P s+Q t+R u=q, \\
S t=w,
\end{aligned}
$$

where $P \in \mathfrak{R}^{(n+m) \times n}, Q \in \mathfrak{R}^{(n+m) \times n}$, and $R \in \mathfrak{R}^{(n+m) \times m}$ are the given matrices, $q \in \mathfrak{R}^{n+m}$ is a given vector,

$$
S:=\left(\begin{array}{cccc}
s_{1} & & & \\
& s_{2} & & \\
& & \ddots & \\
& & & s_{n}
\end{array}\right) \text { is an } n \times n \text { real matrix }
$$

and $w \in \mathfrak{R}^{n}$ is a given nonnegative weighted vector. We denote this problem by the WMLCP. When $w=0$, WMLCP (1) reduces to a mixed linear complementarity problem which has extensively been studied in the literature [2].
In [1], Potra showed that the problem of Fisher market equilibrium may be modeled as a WMLCP, and particularly, it could be solved more efficiently than a corresponding complementarity problem model; Anstreicher [3] proved that the problem of the weighted centering may also be reformulated as a WMLCP and proposed an interior point method to solve it. Since then, this kind of problem has been studied a lot. For example, Potra [4] studied some theories and proposed an interior point method to solve the WMLCP with the involved matrix being sufficient; Zhang [5] presented a smoothing Newton method to solve WMLCPs; Tang [6] proposed a variant nonmonotone smoothing algorithm to solve WMLCPs; Chi et al. [7] investigated the existence and uniqueness of the solution for a class of weighted horizontal linear complementarity problems.

Recall that a standard finite-dimensional complementarity problem (denoted by the CP) is to find a vector pair $(s, t) \in \mathfrak{R}^{2 n}$ such that

$$
\begin{aligned}
s & \geq 0, \\
t & =f(s) \geq 0, \\
s^{\top} t & =0,
\end{aligned}
$$


where $f: \mathfrak{R}^{n} \longrightarrow \mathfrak{R}^{n}$ is a given mapping and the subscript ${ }^{\top}$ denotes transpose. Problem (3) has many applications in fields such as engineering and economics (see, for example, [8]), and it has received great attention [2, 9-11]. Recently, a subclass of CPs, tensor complementarity problems, was also studied extensively (see, for example, survey papers [12-14]). Two extensions of tensor complementarity problems and an application to the problem of traffic equilibrium problems were given in [15].

Inspired by the above papers, we investigate an extension of the CP, called the weighted complementarity problem (denoted by WCP), which is to find a vector pair $(s, t) \in \mathfrak{R}^{2 n}$ such that

$$
\begin{aligned}
s & \geq 0, \\
t & =f(s) \geq 0, \\
S t & =w,
\end{aligned}
$$

where $f: \mathfrak{R}^{n} \longrightarrow \mathfrak{R}^{n}$ is a given mapping. Obviously, when $w=0$, WCP (4) becomes a CP. When $f$ is a linear mapping, say $f(s)=N s+p$ with $N$ being an $n \times n$ real matrix and $p \in \mathfrak{R}^{n}$, the corresponding WCP is named as a weighted linear complementarity problem (WLCP); otherwise, it is named as a weighted nonlinear complementarity problem. Recall that a mapping $f$ is called to be monotone if and only if $(s-t)^{\top}[f(s)-f(t)] \geq 0$ holds for all $s, t \in \mathfrak{R}^{n}$. If $f$ is a monotone mapping, then the corresponding WCP (4) is called a monotone WCP. We will investigate the numerical method for solving the WCP.

It is well known that CP (3) may be reformulated as a system of parameterized smoothing equations in terms of some complementarity function [16-18]. Thus, in order to achieve a solution of CP (3), one may use some Newton-type method to iteratively solve the obtained system of equations and make the smoothing parameter tend to zero. This is the so-called smoothing Newton method. This class of methods has been successfully applied to solving lots of optimization and related problems, including linear complementarity problems [19-24], linear programs [25], nonlinear complementarity problems [26-30], variational inequalities [27, 31], semidefinite complementarity problems [32-37], system of inequalities $[19,34]$, symmetric cone complementarity problems $[35,36]$, mathematical programs with complementarity constraints [38, 39], and absolute value equations [40]. In order to achieve the global convergence, most of the known smoothing Newton methods require the assumption that the solution set of the concerned problem is nonempty and compact. In [29], the author proposed a smoothing Newton method for solving CP (3) with a monotone mapping and showed that the smoothing Newton method is globally convergent when the problem has at least a solution. This assumption is weaker than the ones required in the global convergence of smoothing Newton methods (see, for example, [41]). Moreover, since the nonmonotone line search technique can improve the likelihood of finding a global optimal solution and convergence speed in cases where the involving function is highly nonconvex and has a valley in a small neighbourhood of some point, the nonmonotone line search criterion has been used in some smoothing Newton methods for solving CPs (see, for example, [42-45]). More nonmonotone techniques can be found in [46-50]. In addition, some related Newton-type methods can be found in [51-55].

Inspired by the above methods, we investigate the nonmonotone smoothing Newton method for solving WCP (4). We use the following assumption.

Assumption 1. $f$ is a continuously differentiable monotone mapping and WCP (4) has at least a solution.

In terms of the algorithmic framework of the one studied in [29] for CP (3) and some nonmonotone line search criteria used in $[42,43]$, by using a symmetric perturbed smoothing function, we propose a smoothing Newton method with a mixed line search criterion to solve WCP (4), where, in each iteration, a system of linear equations is solved to find the iterative direction and a line search is performed to achieve the iterative step length. Particularly, we show that the proposed smoothing Newton method is globally convergent if Assumption 1 is satisfied. This assumption is weaker than most of them used in the analysis on the global convergence of smoothing Newton methods for CP (3). When WCP (4) reduces to CP (3), our proof of main results given in this article is simpler than that of the corresponding ones in [29].

The article is organized as follows. In Section 2, we describe a reformulation of WCP (4) and propose a smoothing Newton method for solving WCP (4). In Section 3 , we prove the global convergence of the proposed smoothing Newton method. The preliminary numerical experiments and conclusions are given in Sections 4 and 5, respectively.

In this article, we use $\Re_{+}^{n}$ to represent the nonnegative orthant in $\Re^{n}$ and $\Re_{++}^{n}$ to represent the positive orthant in $\Re^{n}$. Denote

$$
\begin{aligned}
& \Theta:=\{1,2, \ldots, n\}, \\
& \Omega:=\{0,1,2, \ldots\} .
\end{aligned}
$$

For any $s, t \in \Re^{n}, s_{i}$ means the $i$ th component of $s$ and $(s, t)$ stands for $\left(s^{\top}, t^{\top}\right)^{\top}$ simplicity. For any $(\nu, s, t),\left(\nu_{k}, s^{k}, t^{k}\right) \in \mathfrak{R}_{+} \times \mathfrak{R}^{2 n}$, we denote $r:=(\nu, s, t)$ and $r^{k}:=\left(\nu_{k}, s^{k}, t^{k}\right)$. Moreover, the solution set of WCP (4) is denoted by $\mathrm{SOL}_{w}(f)$.

\section{A Smoothing Newton Method}

For any given $\gamma \in \mathfrak{R}$, we define a mapping $\phi_{\gamma}: \mathfrak{R}^{3} \longrightarrow \mathfrak{R}$ by $\phi_{\gamma}(\nu, \alpha, \beta):=(1+\nu)(\alpha+\beta)-\sqrt{(1-\nu)^{2}(\alpha-\beta)^{2}+4 \gamma+4 \nu}$,

for any $(\nu, \alpha, \beta) \in \mathfrak{R}_{+} \times \mathfrak{R} \times \mathfrak{R}$. In fact, when $\gamma=0$, the above mapping (i.e., $\left.\phi_{0}(\cdot, \cdot, \cdot)\right)$ is just the symmetric perturbed smoothing function introduced in [28].

Lemma 1. Given $(\nu, \alpha, \beta, \gamma, \delta) \in \mathfrak{R}_{++} \times \mathfrak{R} \times \mathfrak{R} \times \mathfrak{R}_{+} \times \mathfrak{R}$. Then, we have the following two results:

(a) $\phi_{\gamma}(0, \alpha, \beta)=0$ holds if and only if 


$$
\begin{gathered}
\alpha \geq 0, \\
\beta \geq 0, \\
\alpha \beta=\gamma .
\end{gathered}
$$

(b) $\phi_{\gamma}(\nu, \alpha, \beta)=\delta$ holds if and only if

$$
\begin{gathered}
\alpha+\nu \beta-\frac{\delta}{2}>0, \\
v \alpha+\beta-\frac{\delta}{2}>0, \\
\left(\alpha+\nu \beta-\frac{\delta}{2}\right)\left(\nu \alpha+\beta-\frac{\delta}{2}\right)=\nu+\gamma .
\end{gathered}
$$

Proof. These two results can be easily proved by using the definition of $\phi_{\gamma}(\cdot)$. We omit the proof here.

Furthermore, for any given weighted vector $w \in \mathfrak{R}_{+}^{n}$, we define a mapping for the WCP, $G_{w}: \mathfrak{R}^{1+2 n} \longrightarrow \mathfrak{R}^{1+2 n}$, by

$$
G_{w}(r):=G_{w}(\nu, s, t):=\left(\begin{array}{c}
\nu \\
t-f(s) \\
\Phi_{w}(r)
\end{array}\right),
$$

for any $r:=(\nu, s, t) \in \mathfrak{R} \times \mathfrak{R}^{n} \times \mathfrak{R}^{n}$, where

$$
\Phi_{w}(r):=\left(\begin{array}{c}
\phi_{w_{1}}\left(\nu, s_{1}, t_{1}\right) \\
\phi_{w_{2}}\left(\nu, s_{2}, t_{2}\right) \\
\vdots \\
\phi_{w_{n}}\left(\nu, s_{n}, t_{n}\right)
\end{array}\right) \in \mathfrak{R}^{n} .
$$

Then, by using (9) and Lemma 1 (a), it follows that

$$
G_{w}\left|r=0 \widehat{I} \frac{1}{2}=0,\right| s, t \in \mathrm{SOL}_{w} \mid f .
$$

The following results can be easily obtained.

Proposition 1. Suppose that $f$ is a continuously differentiable monotone mapping. Then,

(a) For each $r \in \mathfrak{R}_{++} \times \Re^{n} \times \Re^{n}$, the mapping $G_{w}$ is continuously differentiable at $r$, and

$$
G_{w}^{\prime}(r)=\left(\begin{array}{ccc}
1 & 0 & 0 \\
0 & -f^{\prime}(s) & I \\
\left(\Phi_{w}(r)\right)_{v}^{\prime} & \left(\Phi_{w}(r)\right)_{s}^{\prime} & \left(\Phi_{w}(r)\right)_{t}^{\prime}
\end{array}\right)
$$

where $I \in \mathfrak{R}^{n \times n}$ is the identity matrix, $v:=$ $\left(\Phi_{w}(r)\right)_{\nu}^{\prime} \in \mathfrak{R}^{n}, \quad U:=\left(\Phi_{w}(r)\right)_{s}^{\prime} \in \mathfrak{R}^{n \times n}$, and $V:=$ $\left(\Phi_{w}(r)\right)_{t}^{\prime} \in \mathfrak{R}^{n \times n}$ with

$$
\begin{aligned}
& v_{i}=s_{i}+t_{i}-\frac{2-(1-v)\left(s_{i}-t_{i}\right)^{2}}{\sqrt{(1-v)^{2}\left(s_{i}-t_{i}\right)^{2}+4 w_{i}+4 v}}, \quad \forall i \in \Theta, \\
& U_{i i}=1+\nu-\frac{(1-v)^{2}\left(s_{i}-t_{i}\right)}{\sqrt{(1-v)^{2}\left(s_{i}-t_{i}\right)^{2}+4 w_{i}+4 v}}, \quad \forall i \in \Theta, \\
& V_{i i}=1+\nu+\frac{(1-v)^{2}\left(s_{i}-t_{i}\right)}{\sqrt{(1-v)^{2}\left(s_{i}-t_{i}\right)^{2}+4 w_{i}+4 v}}, \quad \forall i \in \Theta,
\end{aligned}
$$

and $U_{i j}=0$ and $V_{i j}=0$ for all $i, j \in \Theta$ with $i \neq j$.

(b) $G_{w}^{\prime}(r)$ is nonsingular at each point $r \in \mathfrak{R}_{++} \times \mathfrak{R}^{n} \times \mathfrak{R}^{n}$.

Therefore, by (11) and the continuity of the mapping $G_{w}$, we may find a solution of WCP (4) in the following way: use some Newton-type method to iteratively solve $G_{w}(r)=0$ and make $\left\|G_{w}(r)\right\| \downarrow 0$.

Algorithm 1 (a smoothing Newton method)

Step 0 . Given $w \in \mathfrak{R}_{+}^{n}$. Take $\delta, \sigma \in(0,1), \quad v_{0}>0$, $\left(s^{0}, t^{0}\right) \in \mathfrak{R}^{2 n}$, and a positive integer $M$. Set $r^{0}:=\left(\nu_{0}, s^{0}, t^{0}\right)$. Take $\rho>1$ satisfying $\left\|G_{w}\left(r^{0}\right)\right\| \leq \rho \nu_{0}$. Denote $e^{0}:=(1,0, \ldots, 0) \in \mathfrak{R}^{1+2 n}$. Set $C^{0}:=\left\|G_{w}\left(r^{0}\right)\right\|$ and $Q^{0}:=1$. Choose $\eta_{0} \in[0,1]$ and a sufficiently small positive number $\epsilon$. Set $m_{0}:=1$ and $k:=0$.

Step 1. If $\left\|G_{w}\left(r^{k}\right)\right\|=0$, stop.

Step 2. Let $\mathrm{d} r^{k}:=\left(\mathrm{d} v_{k}, \mathrm{~d} s^{k}, \mathrm{~d} t^{k}\right) \in \mathfrak{R} \times \mathfrak{R}^{n} \times \mathfrak{R}^{n}$ solve

$$
G_{w}\left(r^{k}\right)+G_{w}^{\prime}\left(r^{k}\right) \mathrm{d} r^{k}=\frac{1}{\rho} C^{k} e^{0},
$$

where $G_{w}^{\prime}$ is defined by (4).

Step 3. Let $\gamma_{k}$ be the maximum in $\left\{1, \delta, \delta^{2}, \ldots\right\}$, satisfying

$$
\left\|G_{w}\left(r^{k}+\gamma_{k} \mathrm{~d} r^{k}\right)\right\| \leq\left[1-\sigma\left(1-\frac{1}{\rho}\right) \gamma_{k}\right] C^{k} .
$$

Step 4. Set $m_{k+1}:=\min \{k+1, M\}$. If $\sum_{i=0}^{m_{k}} \eta_{k-i}\left(C^{k-i}-C^{k}\right)>0$, then we set $\eta_{k+1}:=0$; otherwise, we choose $\eta_{k+1} \in(0,1]$. Set

$$
\begin{aligned}
Q^{k+1} & :=1+\eta_{k+1} \sum_{i=0}^{m_{k}} \eta_{k-i}, \\
C^{k+1} & :=\frac{\eta_{k+1} \sum_{i=0}^{m_{k}} \eta_{k-i} C^{k-i}+\left\|G_{w}\left(r^{k}+\gamma_{k} \mathrm{~d} r^{k}\right)\right\|}{Q^{k+1}} .
\end{aligned}
$$

Step 5. Let $r^{k+1}:=r^{k}+\gamma_{k} \mathrm{~d} r^{k}$ and $k:=k+1$. Go to Step 1 .

In Algorithm 1, we set a selection condition: $\sum_{i=0}^{m_{k}} \eta_{k-i}\left(C^{k-i}-C^{k}\right)>0$. If it is satisfied for some $k \in \Omega$, then $\eta_{k+1}=0$; hence, by (16), we have that $C^{k+1}=\left\|G_{w}\left(r^{k+1}\right)\right\|$. In this case, the line search in the $(k+1)$ th iteration is a monotone line search. Moreover, if the above selection condition is not satisfied for some $k \in \Omega$, then the line search in the $(k+1)$ th iteration is a nonmonotone line search. 
Thus, the line search criterion designed in Algorithm 1 is a mixed line search that switches between monotone and nonmonotone line search.

Algorithm 1is new even in the case of the weighted vector involved being the zero vector (in this case, the WCP reduces to the $\mathrm{CP}$ ). Moreover, Algorithm 1 is simpler than many smoothing Newton methods for solving the CP in the sense that it requires only to solve a system of linear equations and to do a line search in each iteration of the algorithm. In fact, a similar algorithmic framework (without the nonmonotone line search) was proposed in [29] for solving (3).

In the following, we show that Algorithm 1 is well defined.

Lemma 2. Suppose that $f$ is a continuously differentiable monotone mapping and the sequence $\left\{r^{k}=\left(\nu_{k}, s^{k}, t^{k}\right)\right\}$ is generated by Algorithm 1. Then, we have the following results:
(a) $C^{k+1} \leq C^{k}$ for all $k \in \Omega$
(b) $C^{k} \leq \rho v_{k}$ for all $k \in \Omega$
(c) $0<v_{k+1} \leq v_{k}$ for all $k \in \Omega$
(d) $\left\|G_{w}\left(r^{k}\right)\right\| \leq C^{k}$ for all $k \in \Omega$
(e) Algorithm 1 is well defined

Proof. (a) For any $k \in \Omega$, if $\sum_{i=0}^{m_{k}} \eta_{k-i}\left(C^{k-i}-C^{k}\right) \leq 0$, then we have that

$$
\begin{aligned}
C^{k+1} & \leq \frac{\eta_{k+1} \sum_{i=0}^{m_{k}} \eta_{k-i} C^{k}+\left\|G_{w}\left(r^{k+1}\right)\right\|}{Q^{k+1}} \\
& \leq \frac{\eta_{k+1} \sum_{i=0}^{m_{k}} \eta_{k-i} C^{k}+\left[1-\sigma(1-(1 / \rho)) \gamma_{k}\right] C^{k}}{Q^{k+1}} \\
& =C^{k}-\frac{\sigma(1-(1 / \rho)) \gamma_{k}}{Q^{k+1}} C^{k} \\
& \leq C^{k},
\end{aligned}
$$

where the first inequality holds due to (16) and the second inequality holds due to (15); otherwise, we have that $\eta_{k+1}=0$, and hence, by (16) and (15), we can obtain that

$$
C^{k+1}=\left\|G_{w}\left(r^{k+1}\right)\right\| \leq\left[1-\sigma\left(1-\frac{1}{\rho}\right) \gamma_{k}\right] C^{k} \leq C^{k} .
$$

Thus, $C^{k+1} \leq C^{k}$ for all $k \in \Omega$.

(b) From Step 0 in Algorithm 1, it is easy to see that $C^{0}=\left\|G_{w}\left(r^{0}\right)\right\| \leq \rho v_{0}$. Suppose that $C^{l} \leq \rho v_{l}$ for some $l \in \Omega$, then

$$
\begin{aligned}
C^{l+1}-\rho \nu_{l+1} & =C^{l+1}-\rho\left[\left(1-\gamma_{l}\right) \nu_{l}+\frac{1}{\rho} \gamma_{l} C^{l}\right] \\
& \leq C^{l+1}-\rho\left[\left(1-\gamma_{l}\right) \frac{1}{\rho} C^{l}+\frac{1}{\rho} \gamma_{l} C^{l}\right] \\
& =C^{l+1}-C^{l} \\
& \leq 0,
\end{aligned}
$$

where the first equality follows from the first equation in (14), the first inequality from the inductive assumption, and the last inequality from the above (a). Thus, $C^{k} \leq \rho v_{k}$ for all $k \in \Omega$.

(c) By the first equation in (14), we have $v_{k+1}=(1-$ $\left.\gamma_{k}\right) \nu_{k}+\gamma_{k}\left(C^{0} / \rho\right)>0$ for all $k \in \Omega$. Moreover, for any $k \in \Omega$, we have that

$$
v_{k+1}=\left(1-\gamma_{k}\right) v_{k}+\frac{1}{\rho} \gamma_{k} C^{k} \leq\left(1-\gamma_{k}\right) v_{k}+\frac{1}{\rho} \gamma_{k} \rho v_{k}=v_{k}
$$

where the first equality follows from the first equation in (14) and the inequality in (b). Thus, $0<v_{k+1} \leq v_{k}$ for all $k \in \Omega$.

(d) By (16), we have that for any $k \in \Omega$,

$$
\begin{aligned}
\left\|G_{w}\left(r^{k}\right)\right\| & =Q^{k} C^{k}-\eta_{k} \sum_{i=1}^{m_{k-1}} \eta_{k-i} C^{k-i} \\
& \leq\left(Q^{k}-\eta_{k} \sum_{i=1}^{m_{k-1}} \eta_{k-i}\right) C^{k}=C^{k},
\end{aligned}
$$

where the inequality follows from the above (a). Thus, $\left\|G_{w}\left(r^{k}\right)\right\| \leq C^{k}$ for all $k \in \Omega$.

(e) On the one hand, since $\nu_{k} \geq 0$ for all $k \in \Omega$, it follows from Proposition 1 that $G_{w}^{\prime}\left(r^{k}\right)$ (see (12)) is invertible, and hence, equation (14) is solvable for all $k \in \Omega$. On the other hand, for any $k \in \Omega$, if we let $R_{w}^{k}(\gamma):=$ $G_{w}\left(r^{k}+\gamma \mathrm{d} r^{k}\right)-G_{w}\left(r^{k}\right)-\gamma G_{w}^{\prime}\left(r^{k}\right) \mathrm{d} r^{k}$, then, by (14), we have that

$$
\begin{aligned}
\left\|G_{w}\left(r^{k}+\gamma \mathrm{d} r^{k}\right)\right\| & =\left\|R_{w}^{k}(\gamma)+G_{w}\left(r^{k}\right)+\gamma G_{w}^{\prime}\left(r^{k}\right) \mathrm{d} r^{k}\right\| \\
& \left.\leq\left\|R_{w}^{k}(\gamma)\right\|+(1-\gamma)\left\|G_{w}\left(r^{k}\right)\right\|+\gamma \frac{1}{\rho}\right] C^{k} \\
& \left.\leq\left\|R_{w}^{k}(\gamma)\right\|+(1-\gamma) C^{k}+\gamma \frac{1}{\rho}\right] C^{k} \\
& \leq\left\|R_{w}^{k}(\gamma)\right\|+\left[1-\gamma\left(1-\frac{1}{\rho}\right)\right] C^{k},
\end{aligned}
$$

where the second inequality holds due to the above (d). Since the function $G$ is continuously differentiable at any $r \in \mathfrak{R}_{++} \times \mathfrak{R}^{n} \times \mathfrak{R}^{n}$, it follows from (a) that $\left\|R_{w}^{k}(\gamma)\right\|=o(\gamma)$ for all $k \in \Omega$. This means that the line search (15) is well defined. Thus, Algorithm 1 is well defined.

\section{Global Convergence of Algorithm 1}

We now show that Algorithm 1 is globally convergent under Assumption 1.

Theorem 1. Let Assumption 1 be satisfied. Then, the following results hold:

(a) The sequence $\left\{r^{k}=\left(\nu_{k}, s^{k}, t^{k}\right)\right\}$ generated by Algorithm 1 is bounded

(b) Every accumulation point of $\left\{\left(s^{k}, t^{k}\right)\right\}$ solves WCP (4) 
Proof. (a) We verify the boundedness of $\left\{r^{k}\right\}$ by the following three parts.

Part 1 . We verify the boundedness of $\left\{v_{k}\right\}$. Such a result holds directly from Lemma 2 (c).

Part 2. We verify the boundedness of $\left\{s^{k}\right\}$. Suppose, on the contrary, that $\left\{s^{k}\right\}$ is unbounded. For our purposes, we need to construct sequences $\left\{x^{k}\right\}$ and $\left\{y^{k}\right\}$ by

$$
\begin{aligned}
x^{k} & :=\frac{1}{v_{k}}\left[t^{k}-f\left(s^{k}\right)\right], \quad \forall k \in \Omega, \\
y^{k} & :=\frac{1}{v_{k}}\left[\Phi_{w}\left(r^{k}\right)+v_{k} s^{k}\right], \quad \forall k \in \Omega .
\end{aligned}
$$

For any $k \in \Omega$, by Lemma 2 (b), we have $C^{k} \leq \rho v_{k}$, and by Lemma 2 (d), we have $\left\|G_{w}\left(r^{k}\right)\right\| \leq C^{k}$. Thus, $\left\|G_{w}\left(r^{k}\right)\right\| \leq \rho v_{k}$ for all $k \in \Omega$. This, together with (23) and (24) as well as the definition of the mapping $G_{w}$ in (9), implies that

$$
\begin{aligned}
\left\|x^{k}\right\| & \leq \sqrt{\rho^{2}-1}, \\
\left\|y^{k}\right\| & \leq \sqrt{\rho^{2}-1},
\end{aligned}
$$

\section{$\forall k \in \Omega$.}

These mean that both sequences $\left\{x^{k}\right\}$ and $\left\{y^{k}\right\}$ are uniformly bounded.

Furthermore, we construct sequences $\left\{\widehat{s}^{k}\right\}$ and $\left\{\hat{t}^{k}\right\}$ by

$$
\left\{\begin{array}{l}
\widehat{s}^{k}:=s^{k}+v_{k} t^{k}-\frac{1}{2} v_{k} y^{k} ; \\
\hat{t}^{k}:=v_{k} s^{k}+t^{k}-\frac{1}{2} v_{k} y^{k},
\end{array} \quad \forall k \in \Omega .\right.
$$

Then, by using Lemma $1(\mathrm{~b})$ and the definition of $y^{k}$ in (24), we have

$$
\begin{aligned}
& \widehat{s}_{i}^{k} \geq 0, \\
& \widehat{t}_{i}^{k} \geq 0, \\
& \widehat{s}_{i}^{k} \hat{t}_{i}^{k}=v_{k}+w_{i}, \\
& \\
& \forall i \in \Theta, \\
& \forall k \in \Omega,
\end{aligned}
$$

which leads to

$$
\left(\widehat{s}^{k}\right)^{\top} \widehat{t}^{k}=n v_{k}+\sum_{i=1}^{n} w_{i}
$$

Moreover, by Assumption 1 it follows that $\mathrm{SOL}_{w}(f) \neq \varnothing$, and hence, there exists $\left(s^{*}, t^{*}\right) \subseteq \mathfrak{R}^{n} \times \mathfrak{R}^{n}$ satisfying

$$
\begin{aligned}
s_{i}^{*} & \geq 0, \\
t_{i}^{*} & =f_{i}\left(s^{*}\right) \geq 0, \\
s_{i}^{*} t_{i}^{*} & =w_{i}, \\
\forall i & \in \Theta .
\end{aligned}
$$

Thus, by using (28) and inequalities given in (27) and (29), we further obtain that

$$
\begin{aligned}
n v_{k} & =\left(\widehat{s}^{k}\right)^{\top} \widehat{t}^{k}-\sum_{i=1}^{n} w_{i} \\
& =\left(\widehat{s}^{k}\right)^{\top} \widehat{t}^{k}-\left(s^{*}\right)^{\top} t^{*} \\
& \geq\left(\widehat{s}^{k}\right)^{\top} \widehat{t}^{k}-\left(s^{*}\right)^{\top} \widehat{t}^{k}-\left(\widehat{s}^{k}\right)^{\top} t^{*}-\left(s^{*}\right)^{\top} t^{*} \\
& =\left(\widehat{s}^{k}-s^{*}\right)^{\top}\left(\widehat{t}^{k}-t^{*}\right), \quad \forall k \in \Omega .
\end{aligned}
$$

Substituting (26) into (30), we obtain that

$$
\begin{aligned}
n v_{k} \geq & \left(s^{k}-s^{*}\right)^{\top}\left(t^{k}-t^{*}\right)+\left(s^{k}-s^{*}\right)^{\top}\left(v_{k} s^{k}-\frac{1}{2} v_{k} y^{k}\right) \\
& +\left(v_{k} t^{k}-\frac{1}{2} v_{k} y^{k}\right)^{\top}\left(t^{k}-t^{*}\right) \\
& +\left(v_{k} t^{k}-\frac{1}{2} v_{k} y^{k}\right)^{\top}\left(v_{k} s^{k}-\frac{1}{2} v_{k} y^{k}\right),
\end{aligned}
$$

holds for any $k \in \Omega$. In what follows, we investigate the right-hand side of (31). Since Assumption 1 holds, we have that the mapping $f$ is monotone, and hence,

$$
\left\{\begin{array}{l}
\left(s^{k}-s^{*}\right)^{\top}\left[f\left(s^{k}\right)-f\left(s^{*}\right)\right] \geq 0, \\
\left(s^{k}-\frac{1}{2} y^{k}\right)^{\top}\left[f\left(s^{k}\right)-f\left(\frac{1}{2} y^{k}\right)\right] \geq 0,
\end{array} \quad \forall k \in \Omega .\right.
$$
$k \in \Omega$,

Hence, by using (23) and (24), we obtain that for any

$$
\begin{aligned}
\left(s^{k}-s^{*}\right)^{\top}\left(t^{k}-t^{*}\right)= & \left(s^{k}-s^{*}\right)^{\top}\left[f\left(s^{k}\right)-f\left(s^{*}\right)\right] \\
& +\left(s^{k}-s^{*}\right)^{\top}\left(v_{k} x^{k}\right) \\
\geq & v_{k}\left(s^{k}-s^{*}\right)^{\top} x^{k},
\end{aligned}
$$

where the first equality holds due to (23) and (29) and

$$
\begin{aligned}
& \left(v_{k} t^{k}-\frac{1}{2} v_{k} y^{k}\right)^{\top}\left(v_{k} s^{k}-\frac{1}{2} v_{k} y^{k}\right) \\
& =v_{k}^{2}\left(s^{k}-\frac{1}{2} y^{k}\right)^{\top}\left[\left(f\left(s^{k}\right)-f\left(\frac{1}{2} y^{k}\right)\right)+\left(f\left(\frac{1}{2} y^{k}\right)-\frac{1}{2} y^{k}+v_{k} x^{k}\right)\right] \\
& \geq v_{k}^{2}\left(s^{k}-\frac{1}{2} y^{k}\right)^{\top}\left[f\left(\frac{1}{2} y^{k}\right)-\frac{1}{2} y^{k}+v_{k} x^{k}\right] .
\end{aligned}
$$

Denote

$$
g\left(s^{k}\right):=\left\|t^{k}-t^{*}\right\|^{2}+\left(t^{*}-\frac{1}{2} v_{k} y^{k}\right)^{\top}\left(t^{k}-t^{*}\right), \quad \forall k \in \Omega .
$$

Then, it holds that either $\left\{g\left(s^{k}\right)\right\}$ is bounded or $g\left(s^{k}\right) \longrightarrow+\infty$ as $k \longrightarrow \infty$. Moreover, we have that 


$$
\left(v_{k} t^{k}-\frac{1}{2} v_{k} y^{k}\right)^{\top}\left(t^{k}-t^{*}\right)=v_{k} g\left(s^{k}\right),
$$

holds for any $k \in \Omega$. Thus, by combining (31), (33), (34), and (36), we obtain that

$$
\begin{aligned}
n \geq & \left(s^{k}-s^{*}\right)^{\top} x^{k}+\left(s^{k}-s^{*}\right)^{\top}\left(s^{k}-\frac{1}{2} y^{k}\right) \\
& +v_{k}\left(s^{k}-\frac{1}{2} y^{k}\right)^{\top}\left[f\left(\frac{1}{2} y^{k}\right)-\frac{1}{2} y^{k}+v_{k} x^{k}\right]+g\left(s^{k}\right),
\end{aligned}
$$

$\forall k \in \Omega$.

By the assumption that $\left\{s^{k}\right\}$ is unbounded, it holds that the right-hand side of (37) tends to $+\infty$ as $k \longrightarrow+\infty$, which is a contradiction to the fact that the left-hand side of (37) is a constant. Therefore, the assumption that $\left\{s^{k}\right\}$ is unbounded does not hold, i.e., $\left\{s^{k}\right\}$ is bounded.

Part 3. We verify the boundedness of $\left\{t^{k}\right\}$. On the one hand, we have that $\left\{f\left(s^{k}\right)\right\}$ is bounded since $\left\{s^{k}\right\}$ is bounded and the mapping $f$ is continuous. On the other hand, by (23), we have that $t^{k}=v_{k} x^{k}+f\left(s^{k}\right)$ holds for all $k \in \Omega$. Thus, by the boundedness of sequences $\left\{v_{k}\right\},\left\{x^{k}\right\}$, and $\left\{f\left(s^{k}\right)\right\}$, we can obtain that $\left\{t^{k}\right\}$ is bounded. Therefore, by combining Part 1 with Part 2 and Part 3, we obtain that $\left\{r^{k}\right\}$ is bounded.

(b) We verify that every accumulation point of $\left\{\left(s^{k}, t^{k}\right)\right\}$ solves WCP (3). From Lemma 2 (a)-(d), we have that for all $k \in \Omega$,

$$
0 \leq\left\|G_{w}\left(r^{k+1}\right)\right\| \leq C^{k+1} \leq C^{k} \leq \rho v_{k} \leq \rho v_{0}<\infty .
$$

Thus, by using the boundedness of the iterative sequence in (a) and taking the subsequence if necessary, we can assume that $r^{*}:=\left(\nu_{*}, s^{*}, t^{*}\right)$ is the limiting point of the sequence $\left\{r^{k}\right\}$ and denote that $C^{*}:=\operatorname{limit}_{k \rightarrow \infty} C^{k}$ and $G_{w}^{*}:=\operatorname{limit}_{k \longrightarrow \infty}\left\|G_{w}\left(r^{k}\right)\right\|$. Since the mapping $G_{w}$ is continuous, it is obvious that $G_{w}^{*}=\left\|G_{w}\left(r^{*}\right)\right\|$. Moreover, it is not difficult to verify from (16) that $C^{*}=G_{w}^{*}$. If $G_{w}^{*}=0$, then we can derive the desired result by a simple continuity discussion.

In the following, assume $G_{w}^{*} \neq 0$. Then, $C^{*}=G_{w}^{*}>0$ and $\nu_{*}>0$. We consider the following two cases.

Case 1. Suppose that $\gamma_{k} \geq \widehat{\gamma}>0$ for all $k \in \Omega$, where $\widehat{\gamma}$ is an $s$ constant. Then, by (15), we have that for any $k \in \Omega$,

$$
\begin{aligned}
\left\|G_{w}\left(r^{k}+\gamma_{k} \mathrm{~d} r^{k}\right)\right\| & \leq\left[1-\sigma\left(1-\frac{1}{\rho}\right) \gamma_{k}\right] C^{k} \\
& \leq\left[1-\sigma\left(1-\frac{1}{\rho}\right) \widehat{\gamma}\right] C^{k} .
\end{aligned}
$$

Let $k \longrightarrow \infty$, so we can obtain that $1 \leq 1-\sigma(1-(1 / \rho)) \widehat{\gamma}$, which leads to a contradiction.

Case 2. Suppose that $\operatorname{limit}_{k \longrightarrow \infty} \gamma_{k}=0$. Then, for any sufficiently large $k \in \Omega$, the stepsize $\bar{\gamma}_{k}:=\left(\gamma_{k} / \delta\right)$ does not satisfy the line search criteria (15), i.e.,

$$
\begin{aligned}
\left\|G_{w}\left(r^{k}+\bar{\gamma}_{k} \mathrm{~d} r^{k}\right)\right\| & >\left[1-\sigma\left(1-\frac{1}{\rho}\right) \bar{\gamma}_{k}\right] C^{k} \\
& \geq\left[1-\sigma\left(1-\frac{1}{\rho}\right) \bar{\gamma}_{k}\right]\left\|G_{w}\left(r^{k}\right)\right\|,
\end{aligned}
$$

where the second inequality holds from Lemma 2 (d). Thus, for any sufficiently large $k \in \Omega$,

$$
\frac{\left[\left\|G_{w}\left(r^{k}+\bar{\gamma}_{k} \mathrm{~d} r^{k}\right)\right\|-\left\|G_{w}\left(r^{k}\right)\right\|\right]}{\bar{\gamma}_{k}}>-\sigma\left(1-\frac{1}{\rho}\right)\left\|G_{w}\left(r^{k}\right)\right\| .
$$

Since $\nu_{*}>0$, it is easy to show from (14) that the sequence $\left\{\mathrm{d} r^{k}\right\}$ is convergent. Denote $\mathrm{d} r^{*}:=\operatorname{limit}_{k \longrightarrow \infty} \mathrm{d} r^{k}$. Let $k \longrightarrow \infty$, and it follows from (41) that

$$
\begin{aligned}
-\sigma\left(1-\frac{1}{\rho}\right) G_{w}^{*} & \leq \frac{1}{G_{w}^{*}}\left\langle G_{w}\left(r^{*}\right), G_{w}^{\prime}\left(r^{*}\right) \mathrm{d} r^{*}\right\rangle \\
& =-G_{w}^{*}+\frac{C^{*}}{\rho G_{w}^{*}}\left\langle G_{w}\left(r^{*}\right), e^{0}\right\rangle \\
& \leq-G_{w}^{*}+\frac{C^{*}}{\rho}
\end{aligned}
$$

which leads to $-\sigma(1-(1 / \rho)) \leq-1+(1 / \rho)$. This is a contradiction.

Therefore, by combining Case 1 with Case 2, we obtain that every accumulation point of $\left\{\left(s^{k}, t^{k}\right)\right\}$ solves WCP (4).

Combining (a) with (b), we complete the proof of the theorem.

The basic idea in showing the boundedness of the iterative sequence (i.e., the proof of Theorem 1 (a)) is similar to the ones in [29], Lemma 3.4 and Theorem 3.1. However, the proof of Theorem 1 is more simpler than those of Lemma 3.4 and Theorem 3.1 in [29].

\section{Numerical Experiments}

In this section, we implement Algorithm 1 for solving weighted linear complementarity problems. All experiments were performed on a Thinkpad notebook computer with $2.5 \mathrm{GHz} \mathrm{CPU}$ and $8 \mathrm{~GB}$ memory. The codes are run in MATLAB R2016a under Win 10. Our experiments are divided into two parts.

Part 1. In this part, we implement Algorithm 1 for solving WCP (4). Specifically, we test the following problem:

Problem 1. Consider the following WLCP:

$$
\begin{aligned}
s & \geq 0, \\
t & =N s+p, \\
S t & =w,
\end{aligned}
$$

which is randomly generated in the following way: for any given positive integer $n$, take $N:=A^{\top} A$ with $A:=0.1 * \operatorname{rand}(100, n) \quad$ and $\quad s^{*}:=\operatorname{rand}(n, 1)$. Let 
$\Lambda:=\operatorname{randi}(n, 0.5 * n, 1)$, and take $p$ in the following way: $p_{i}:=-\left(N s^{*}\right)_{i}$ for all $i \in \Lambda$ and $p_{i}:=\operatorname{rand}(1,1)$ for all $i \in\{1,2, \ldots, n\} \backslash \Lambda$. Let $w:=S^{*} t^{*}$.

Obviously, this problem is a monotone WLCP, which is solvable. In this problem, $w_{i}=0$ for all $i \in \Lambda$. In the experiments, we take

$$
\begin{aligned}
M & :=5, \\
\delta & :=0.5, \\
\sigma & :=0.001, \\
\nu_{0} & :=0.01 .
\end{aligned}
$$

Suppose $n$ is taken as those listed in Table 1. Take the starting point $s^{0}:=-0.5 * \operatorname{rand}(n, 1)$. Set $t^{0}:=N s^{0}+p$ and $r^{0}:=\left(v_{0}, s^{0}, t^{0}\right)$. Take $\rho:=\left(\left\|G_{w}\left(r^{0}\right)\right\| / \nu_{0}\right)$. We take $\eta_{0}:=0.8$, and for any $k \in \Omega$, if the condition $\sum_{i=0}^{m_{k}} \eta_{k-i}\left(C^{k-i}-C^{k}\right)>0$ is satisfied, we set $\eta_{k+1}:=0$; otherwise, we set $\eta_{k+1}:=\min \left(0.01,0.5 \eta_{k}\right)$. We terminate Algorithm 1 when $\left\|G_{w}\left(r^{k}\right)\right\| \leq 10^{-6}$.

For every choice of $n$, the corresponding problem is tested five times and the experimental results are reported in Table 1, where It denotes the number of iterations, Itm denotes the number of iterations when the monotone line search is used (i.e., the case of $\eta_{k}=0$ ), NF denotes the number of evaluations for the mapping $G_{w}\left(r^{k}\right)$, Val denotes the value of $\left\|G_{w}\left(r^{k}\right)\right\|$, and Cpu denotes the CPU time in seconds, respectively.

From Table 1, we have the following observations:

(i) From columns "It" and "Itm" in Table 1, we see that the monotone line search and the nonmonotone line search both work in Algorithm 1. So, this algorithm is really an algorithm with a mixed line search.

(ii) It can be seen that Algorithm 1 is effective for solving WLCPs since each tested problem was successfully solved in few iterations and short CPU time.

(iii) Algorithm 1 is robust since different tested problems having the same size were successfully solved with small differences among the numbers of iterations.

Part 2. In fact, Algorithm 1 can be applied to solve WMLCP (1). For this purpose, instead of the mapping $G_{w}(\cdot)$ defined by (9), we define a mapping for the WMLCP, $G_{w}: \mathfrak{R}^{1+2 n+m} \longrightarrow \mathfrak{R}^{1+2 n+m}$, by

$$
G_{w}(r):=G_{w}(\nu, s, t, u):=\left(\begin{array}{c}
v \\
P s+Q t+R u-q \\
\Phi_{w}(r)
\end{array}\right)
$$

for any $r:=(\nu, s, t, u) \in \mathfrak{R} \times \mathfrak{R}^{n} \times \mathfrak{R}^{n} \times \mathfrak{R}^{m}$, where the mapping $\Phi_{w}$ is the same as the one defined in Section 2 and $G_{w}^{\prime}(r)$ is used to denote the Jacobian matrix of $G_{w}(\cdot)$ at any $r=(\nu, s, t, u) \in \mathfrak{R} \times \mathfrak{R}^{n} \times \mathfrak{R}^{n} \times \mathfrak{R}^{m}$.

In this part, we implement Algorithm 1 for solving this type of weighted complementarity problem and compare it
TABle 1: The numerical results of Problem 1.

\begin{tabular}{lccccc}
\hline$n$ & It & Itm & NF & Val & Cpu \\
\hline \multirow{4}{*}{1000} & 7 & 5 & 13 & $9.07 e-07$ & $1.94 e+00$ \\
& 8 & 5 & 20 & $1.00 e-07$ & $3.08 e+00$ \\
& 7 & 5 & 15 & $1.17 e-07$ & $2.09 e+00$ \\
& 6 & 5 & 8 & $4.23 e-07$ & $1.69 e+00$ \\
& 8 & 5 & 13 & $3.55 e-07$ & $2.22 e+00$ \\
\hline \multirow{5}{*}{3000} & 10 & 5 & 29 & $5.69 e-07$ & $3.56 e+01$ \\
& 9 & 8 & 22 & $2.75 e-09$ & $3.30 e+01$ \\
& 9 & 5 & 24 & $7.07 e-07$ & $3.28 e+01$ \\
& 10 & 5 & 26 & $8.09 e-08$ & $3.60 e+01$ \\
& 11 & 5 & 28 & $4.99 e-07$ & $4.57 e+01$ \\
\hline \multirow{5}{*}{5000} & 10 & 9 & 26 & $1.12 e-07$ & $1.50 e+02$ \\
& 10 & 5 & 26 & $1.21 e-07$ & $1.50 e+02$ \\
& 11 & 5 & 27 & $1.11 e-08$ & $1.63 e+02$ \\
& 10 & 5 & 27 & $1.49 e-07$ & $1.66 e+02$ \\
& 10 & 5 & 25 & $9.83 e-08$ & 158 \\
\hline
\end{tabular}

with two algorithms studied in the literature. The test problem is constructed in a similar way as those in $[5,6]$. Specifically, we test the following problem.

Problem 2. Consider WMLCP (1), where matrices $P, Q$, and $R$ and vector $q$ are generated as follows:

$$
\begin{gathered}
P=\left(\begin{array}{c}
A \\
M
\end{array}\right), \\
Q=\left(\begin{array}{c}
0_{m n} \\
-I_{n}
\end{array}\right), \\
R=\left(\begin{array}{c}
0_{m m} \\
-A^{\top}
\end{array}\right), \\
q=\left(\begin{array}{c}
b \\
-f
\end{array}\right),
\end{gathered}
$$

where $A:=\left(I_{m}-B\right) \in \mathfrak{R}^{m \times n}$ with $B:=0.05 * \operatorname{rand}(m, n-m)$ and $I_{m}$ being an $m \times m$ identity matrix, $M:=\operatorname{diag}(\operatorname{rand}(n, 1)), 0_{m n}$ is an $m \times n$ matrix with all entries being zero, $b:=A s^{*}$ with $s^{*}:=0.5 * \operatorname{rand}(n, 1)$, and $f:=0.5 * \operatorname{rand}(n, 1) . \quad$ Moreover, $\quad w:=S^{*} t^{*} \quad$ with $t^{*}:=M * s^{*}+f$.

In order to generate a strictly feasible starting point, we first take stemp $=\left[s_{I} ; s_{B}\right]$ with $s_{B}:=0.1 * \operatorname{rand}(n-m, 1)$ and $s_{I}:=B * s_{B}$, and then, we set the starting point by

$$
\begin{aligned}
& s^{0}:=0.5 * \text { stemp }+s^{*}, \\
& t^{0}:=M * s^{0}+f, \\
& y^{0}:=\operatorname{zeros}(m, 1) .
\end{aligned}
$$

We implement our algorithm (i.e., Algorithm 1), Algorithm 1 in [5], and Algorithm 1 in [1] for solving Problem 2 , respectively. For our algorithm, all parameters are selected as the same as those in experiments of Part 1; and for Algorithm 1 in [5], all parameters are selected as the same as those in experiments in [5]. Denote 
Table 2: The numerical results of Problem 2.

\begin{tabular}{|c|c|c|c|c|}
\hline$m$ & $n$ & $\begin{array}{c}\text { Algorithm } 1 \\
\text { It/Cpu/Val }\end{array}$ & $\begin{array}{c}\text { Algorithm } 1 \text { in [5] } \\
\text { It/Cpu/Val }\end{array}$ & $\begin{array}{c}\text { Algorithm } 1 \text { in [1] } \\
\text { It/Cpu/Val }\end{array}$ \\
\hline 1000 & 1500 & $\begin{array}{l}8 / 5.41 e+00 / 5.30 e-07 \\
9 / 5.64 e+00 / 5.32 e-08 \\
8 / 4.58 e+00 / 7.84 e-07 \\
8 / 3.84 e+00 / 6.81 e-08 \\
8 / 4.22 e+00 / 2.96 e-08\end{array}$ & $\begin{array}{l}8 / 4.56 e+00 / 7.04 e-07 \\
9 / 4.78 e+00 / 7.03 e-09 \\
8 / 4.66 e+00 / 2.68 e-08 \\
8 / 4.67 e+00 / 6.36 e-10 \\
9 / 4.89 e+00 / 8.51 e-09\end{array}$ & $\begin{array}{c}19 / 1.85 e+01 / 1.64 e-07 \\
20 / 2.00 e+01 / 1.25 e-07 \\
15 / 1.41 e+01 / 1.10 e-07 \\
18 / 1.75 e+01 / 2.19 e-07 \\
17 / 18 / 5.41 e-07\end{array}$ \\
\hline 1500 & 2000 & $\begin{array}{c}9 / 1.19 e+01 / 6.63 e-08 \\
10 / 1.35 e+01 / 2.51 e-07 \\
8 / 1.07 e+01 / 1.22 e-07 \\
10 / 1.31 e+01 / 2.76 e-08 \\
9 / 1.23 e+01 / 6.89 e-08\end{array}$ & $\begin{array}{c}9 / 1.13 e+01 / 3.42 e-10 \\
12 / 1.60 e+01 / 6.32 e-09 \\
9 / 1.23 e+01 / 1.61 e-08 \\
10 / 1.26 e+01 / 4.22 e-09 \\
10 / 1.28 e+01 / 7.38 e-08\end{array}$ & $\begin{array}{l}21 / 5.45 e+01 / 2.53 e-07 \\
23 / 5.53 e+01 / 5.90 e-07 \\
22 / 5.48 e+01 / 8.99 e-07 \\
22 / 5.32 e+01 / 2.85 e-07 \\
20 / 4.80 e+01 / 9.96 e-07\end{array}$ \\
\hline 2500 & 3000 & $\begin{array}{c}10 / 5.18 e+01 / 1.65 e-08 \\
8 / 4.13 e+01 / 7.81 e-07 \\
10 / 4.80 e+01 / 1.03 e-09 \\
9 / 4.29 e+01 / 1.82 e-08 \\
9 / 4.53 e+01 / 7.03 e-07\end{array}$ & $\begin{array}{l}12 / 5.68 e+01 / 5.01 e-07 \\
10 / 4.96 e+01 / 1.50 e-10 \\
12 / 5.99 e+01 / 3.24 e-08 \\
12 / 5.68 e+01 / 1.78 e-07 \\
10 / 4.86 e+01 / 9.47 e-08\end{array}$ & $\begin{array}{l}33 / 2.92 e+02 / 8.33 e-07 \\
26 / 2.34 e+02 / 2.16 e-07 \\
39 / 3.63 e+02 / 6.98 e-07 \\
35 / 3.11 e+02 / 1.07 e-07 \\
30 / 2.71 e+02 / 2.00 e-07\end{array}$ \\
\hline
\end{tabular}

$$
\begin{aligned}
\text { Res } & =\|P s+Q t+R u\|_{\infty}, \\
\text { Gap } & =\|w-S x\|_{\infty}, \\
\operatorname{norm} G & =\left\|G_{w}\left(r^{k}\right)\right\| .
\end{aligned}
$$

For the first two algorithms, the termination criterion is $\max ($ Res, Gap, norm $G) \leq 10^{-6}$; while for the last algorithm, the termination criterion is $\max ($ Res, Gap $) \leq 10^{-6}$.

In the experiments of each algorithm, for every choice of $n$ and $m$, the corresponding problem is tested five times. The experimental results are reported in Table 2, where It and Cpu denote the number of iterations and the CPU time in seconds, respectively, and Val denotes $\max ($ Res, Gap, norm $G$ ) for the first two algorithms and $\max$ (Res, Gap) for the last algorithm.

From the numerical results in Table 2, it is easy to see that our algorithm is comparable to Algorithm 1 in [5], and these two algorithms are more efficient than Algorithm 1 in [1].

\section{Conclusions}

We proposed a smoothing Newton method with a mixed line search for solving monotone WCPs and showed the global convergence of the method under a weak assumption. The preliminary experimental results demonstrate the effectiveness and robustness of the method for solving the monotone WCP. We believe that many other smoothing Newton methods can also be modified to solve the WCP. Two future issues which have to be investigated are the theoretical properties of WCPs and solution methods for large-scale WCPs.

\section{Data Availability}

No data were used to support this study.

\section{Conflicts of Interest}

The authors declare that they have no conflicts of interest.

\section{Acknowledgments}

This work was partially supported by the Science Fund of Educational Commission of Hubei Province in China (no. B2015335) and the Science Fund of Wuhan Technology and Business University (no. A2014024).

\section{References}

[1] F. A. Potra, "Weighted complementarity problems-a new paradigm for computing equilibria," SIAM Journal on Optimization, vol. 22, no. 4, pp. 1634-1654, 2012.

[2] R. W. Cottle, J. S. Pang, and R. E. Stone, The Linear Complementarity Problem, Academic Press, Boston, MA, USA, 1992.

[3] K. M. Anstreicher, "Interior-point algorithms for a generalization of linear programming and weighted centering," Optimization Methods and Software, vol. 27, no. 4-5, pp. 605-612, 2012.

[4] F. A. Potra, "Sufficient weighted complementarity problems," Computational Optimization and Applications, vol. 64, no. 2, pp. 467-488, 2016.

[5] J. Zhang, "A smoothing Newton algorithm for weighted linear complementarity problem," Optimization Letters, vol. 10, no. 3, pp. 499-509, 2016.

[6] J. Tang, "A variant nonmonotone smoothing algorithm with improved numerical results for large-scale LWCPs," Computational and Applied Mathematics, vol. 37, no. 3, pp. 3927-3936, 2018.

[7] X. Chi, M. S. Gowda, and J. Tao, "The weighted horizontal linear complementarity problem on a Euclidean Jordan algebra," Journal of Global Optimization, vol. 73, no. 1, pp. 153-169, 2019.

[8] M. C. Ferris and J. S. Pang, "Engineering and economic applications of complementarity problems," SIAM Review, vol. 39, no. 4, pp. 669-713, 1997.

[9] F. Facchinei and J. S. Pang, "Finite-dimemsional variational inequalities and complementarity problems,"Operations Research, vol. 1, Springer-Verlag, New York, NY, USA, 2003.

[10] F. Facchinei and J. S. Pang, "Finite-dimemsional variational inequalities and complementarity problems,"Operations Research, vol. 2, Springer-Verlag, New York, NY, USA, 2003. 
[11] J. Han, N. Xiu, and H. D. Qi, Nonlinear Complementarity Theory and Algorithms, Shanghai Science and Technology Press, Shanghai, China, 2006, in Chinese.

[12] Z.-H. Huang and L. Qi, "Tensor complementarity problems-part I: basic theory," Journal of Optimization Theory and Applications, vol. 183, no. 1, pp. 1-23, 2019.

[13] L. Qi and Z.-H. Huang, "Tensor complementarity problems-part II: solution methods," Journal of Optimization Theory and Applications, vol. 183, no. 2, pp. 365-385, 2019.

[14] Z.-H. Huang and L. Qi, "Tensor complementarity problems-part III: applications," Journal of Optimization Theory and Applications, vol. 183, no. 3, pp. 771-791, 2019.

[15] Y. Xu, W. Gu, and H. Huang, "Solvability of two classes of tensor complementarity problems," Mathematical Problems in Engineering, vol. 2019, Article ID 6107517, 8 pages, 2019.

[16] D. Sun and L. Qi, "On NCP-functions," Computational Optimization and Applications, vol. 13, no. 1-3, pp. 201-220, 1999.

[17] S.-L. Hu, Z.-H. Huang, and J.-S. Chen, "Properties of a family of generalized NCP-functions and a derivative free algorithm for complementarity problems," Journal of Computational and Applied Mathematics, vol. 230, no. 1, pp. 69-82, 2009.

[18] J. Sun, X.-R. Wu, B. Saheya, J.-S. Chen, and C.-H. Ko, "Neural network for solving SOCQP and SOCCVI based on two discrete-type classes of SOC complementarity functions," Mathematical Problems in Engineering, vol. 2019, Article ID 4545064, 18 pages, 2019.

[19] C. Chen and O. L. Mangasarian, "Smoothing methods for convex inequalities and linear complementarity problems," Mathematical Programming, vol. 71, no. 1, pp. 51-69, 1995.

[20] C. Kanzow, "Some noninterior continuation methods for linear complementarity problems," SIAM Journal on Matrix Analysis and Applications, vol. 17, no. 4, pp. 851-868, 1996.

[21] J. Burke and S. Xu, "A non-interior predictor-corrector path following algorithm for the monotone linear complementarity problem," Mathematical Programming, vol. 87, no. 1, pp. 113-130, 2000.

[22] X. Chen and Y. Ye, "On smoothing methods for the $P_{0}$ matrix linear complementarity problem," SIAM Journal on Optimization, vol. 11, no. 2, pp. 341-363, 2000.

[23] Y. B. Zhao and D. Li, "A globally and locally superlinearly convergent non-interior-point algorithm for $P_{0}$ LCPs," SIAM Journal on Optimization, vol. 13, no. 4, pp. 1196-1221, 2003.

[24] Z.-H. Huang, L. Qi, and D. Sun, "Sub-quadratic convergence of a smoothing Newton algorithm for the $P_{0^{-}}$and monotone LCP," Mathematical Programming, vol. 99, no. 3, pp. 423-441, 2004.

[25] S. Engelke and C. Kanzow, "Improved non-interior continuation methods for the solution of linear programming," Numerische Mathematik, vol. 90, no. 3, pp. 487-507, 2002.

[26] B. Chen and N. Xiu, "A global linear and local quadratic noninterior continuation method for nonlinear complementarity problems based on Chen-Mangasarian smoothing functions," SIAM Journal on Optimization, vol. 9, no. 3, pp. 605-623, 1999.

[27] L. Qi, D. Sun, and G. Zhou, “A new look at smoothing Newton methods for nonlinear complementarity problems and box constrained variational inequality problems," Mathematical Programming, vol. 87, no. 1, pp. 1-35, 2000.

[28] Z. H. Huang, J. Han, and Z. Chen, "A predictor-corrector smoothing Newton method, based on a new smoothing function, for solving the NCP with a $P_{0}$ function," Journal of Optimization Theory and Applications, vol. 117, no. 1, pp. 39-68, 2003.
[29] Z.-H. Huang, "Locating a maximally complementary solution of the monotone NCP by using non-interior-point smoothing algorithms," Mathematical Methods of Operations Research, vol. 61, no. 1, pp. 41-55, 2005.

[30] Z.-H. Huang and L. Qi, "Formulating an $n$-person noncooperative game as a tensor complementarity problem," Computational Optimization and Applications, vol. 66, no. 3, pp. 557-576, 2017.

[31] H.-D. Qi, “A regularized smoothing Newton method for box constrained variational inequality problems with $P_{0}$-functions," SIAM Journal on Optimization, vol. 10, no. 2, pp. 315-330, 2000.

[32] X. Chen and P. Tseng, "Non-interior continuation methods for solving semidefinite complementarity problems," Mathematical Programming, vol. 95, no. 3, pp. 431-474, 2003.

[33] Z. Huang and J. Han, "Non-interior continuation method for solving the monotone semidefinite complementarity problem," Applied Mathematics and Optimization, vol. 47, no. 3, pp. 195-211, 2003.

[34] Z. H. Huang, Y. Zhang, and W. Wu, "A smoothing-type algorithm for solving system of inequalities," Journal of Computational and Applied Mathematics, vol. 220, no. 1-2, pp. 355-363, 2008.

[35] L. Kong, J. Sun, and N. Xiu, "A regularized smoothing Newton method for symmetric cone complementarity problems," SIAM Journal on Optimization, vol. 19, no. 3, pp. 1028-1047, 2008.

[36] Z.-H. Huang and T. Ni, "Smoothing algorithms for complementarity problems over symmetric cones," Computational Optimization and Applications, vol. 45, no. 3, pp. 557-579, 2010.

[37] N. Lu and Z.-H. Huang, "A smoothing Newton algorithm for a class of non-monotonic symmetric cone linear complementarity problems," Journal of Optimization Theory and Applications, vol. 161, no. 2, pp. 446-464, 2014.

[38] Z.-H. Huang and J. Sun, "A smoothing Newton algorithm for mathematical programs with complementarity constraints," Journal of Industrial \& Management Optimization, vol. 1, no. 2, pp. 153-170, 2005.

[39] Y. Chen and Z. Wan, "A new smoothing method for mathematical programs with complementarity constraints based on logarithm-exponential function," Mathematical Problems in Engineering, vol. 2018, Article ID 5056148, 11 pages, 2018.

[40] X. Jiang and Y. Zhang, "A smoothing-type algorithm for absolute value equations," Journal of Industrial \& Management Optimization, vol. 9, no. 4, pp. 789-798, 2013.

[41] Z. Huang, "Sufficient conditions on nonemptiness and boundedness of the solution set of the $P_{0}$ function nonlinear complementarity problem," Operations Research Letters, vol. 30, no. 3, pp. 202-210, 2002.

[42] Z. Huang, S. Hu, and J. Han, "Convergence of a smoothing algorithm for symmetric cone complementarity problems with a nonmonotone line search," Science in China Series A: Mathematics, vol. 52, no. 4, pp. 833-848, 2009.

[43] S.-L. Hu, Z.-H. Huang, and P. Wang, "A nonmonotone smoothing Newton algorithm for solving nonlinear complementarity problems," Optimization Methods and Software, vol. 24, no. 3, pp. 447-460, 2009.

[44] T. Ni and P. Wang, "A smoothing-type algorithm for solving nonlinear complementarity problems with a non-monotone line search," Applied Mathematics and Computation, vol. 216, no. 7, pp. 2207-2214, 2010. 
[45] J. Zhu, H. Liu, and C. Liu, "A family of new smoothing functions and a nonmonotone smoothing Newton method for the nonlinear complementarity problems," Journal of Applied Mathematics and Computing, vol. 37, no. 1-2, pp. 647-662, 2011.

[46] T. Dehghan Niri, M. Heydari, and M. M. Hosseini, "An improvement of adaptive cubic regularization method for unconstrained optimization problems," International Journal of Computer Mathematics, pp. 1-17, 2020.

[47] T. Dehghan Niri, M. Heydari, and M. M. Hosseini, "Correction of trust region method with a new modified Newton method," International Journal of Computer Mathematics, vol. 97, no. 5, pp. 1118-1132, 2020.

[48] S. Huang, Z. Wan, and J. Zhang, "An extended nonmonotone line search technique for large-scale unconstrained optimization," Journal of Computational and Applied Mathematics, vol. 330, pp. 586-604, 2018.

[49] S. Huang and Z. Wan, "A new nonmonotone spectral residual method for nonsmooth nonlinear equations," Journal of Computational and Applied Mathematics, vol. 313, pp. 82101, 2017.

[50] S. Huang, Z. Wan, and X. Chen, "A new nonmonotone line search technique for unconstrained optimization," Numerical Algorithms, vol. 68, no. 4, pp. 671-689, 2015.

[51] G. Q. Wang, Y. J. Yue, and X. Z. Cai, "A weighted-pathfollowing method for monotone horizontal linear complementarity problem," Advances in Soft Computing, Springer, Berlin, Germany, pp. 479-487, 2009.

[52] F. Gurtuna, C. Petra, F. A. Potra, O. Shevchenko, and A. Vancea, "Corrector-predictor methods for sufficient linear complementarity problems," Computational Optimization and Applications, vol. 48, no. 3, pp. 453-485, 2011.

[53] T. Dehghan Niri, S. A. Shahzadeh Fazeli, and M. Heydari, "A two-step improved Newton method to solve convex unconstrained optimization problems," Journal of Applied Mathematics and Computing, vol. 62, no. 1-2, pp. 37-53, 2020.

[54] T. Dehghan Niri, M. M. Hosseini, and M. Heydari, "An efficient improvement of the Newton method for solving nonconvex optimization problems," Computational Methods for Differential Equations, vol. 7, no. 1, pp. 69-85, 2019.

[55] T. Dehghan Niri, M. M. Hosseini, and M. Heydari, "A new modified trust region algorithm for solving unconstrained optimization problems," Journal of Mathematical Extension, vol. 12, no. 4, pp. 115-135, 2018. 